\title{
X-RAYS FROM HOT STARS AND THE ROLE OF RELATIVISTIC ELECTRONS
}

\author{
A.M.T.Pollock \\ Computer \& Scientific Co. Ltd., Sheffield S11 7EY, England
}

This paper challenges the usual view that $X$-ray emission from hot stars is direct thermal radiation from shocks in the wind. Instead, detailed calculations show that the relativistic electrons in the wind required to explain the non thermal radio flux of some hot stars can also account for the $X$-rays from all single hot stars via inverse Compton scattering of photospheric radiation. First, there are two essential general points to understand; (1) the heavily absorbed accreting neutron-star X-ray sources orbiting OB companions in binary systems like $4 \mathrm{U} 1700-37$ and Vela X-1 show without doubt that the instrinsic X-rays from hot stars cannot originate in a corona or any other structure confined close to the photosphere; and (2) the observed 4:1 ratio of mean WN:WC X-ray luminosities, much less than the value 50:1 expected for a point source, strongly implies a distributed X-ray source. The non thermal radio components observed by Abbott and others show that relativistic electrons are present far out in the wind of some and perhaps all hot stars. The single most important reason for supposing that these same electrons also account for the ubiquitous $\mathrm{X}$-rays is that the radiation density is so high that Compton losses cause very rapid cooling. It is complicated to work out in any detail the observational consequences of relativistic electrons in a stellar wind because (1) electrons cool via ionisation, adiabatic, synchrotron, inverse-Compton and Bremsstrahlung energy losses of which only the final three give potentially observable radiation at radio, $\mathrm{X}$-ray and $\gamma$-ray frequencies respectively; and (2) only electrons in the outer parts of the wind are visible because of free-free radio absorption and photoelectric $\mathrm{X}$-ray absorption. The relative importance of the different energydependent cooling processes changes as electrons are advected out with the wind. Near the star, ionisation losses cut off the supply of observable low-energy electrons until Compton losses take over at electron $\gamma \mathrm{s}$ of $70-80$ in the W-R stars and 20-30 in the OB stars . Further out adiabatic losses are the principal low-energy cooling mechanism before giving way to Compton losses at an electron break energy that increases linearly with radius. Synchrotron and Bremsstrahlung losses are always small. The solution of the transport equation for some supposed electron injection law tempered by these energy-loss mechanisms gives the radial evolution of the electron spectrum that, in turn, allows local radiative emissivities to be calculated. These then combine with the local optical depths to determine how much radiation escapes to the observer. Most of the 20 or so stellar parameters involved in the calculations are reasonably well known. The exceptions are the surface magnitude, $B_{*}$, and geometry of the stellar magnetic field and how the electrons are injected. Solving the 1-D electron transport equation with a Lax-Wendroff upwind differencing scheme before numerical integration of the emissivity formulae leads to the following main conclusions and predictions: (1) a small population of relativistic electrons can reproduce simultaneously the observed non thermal radio fluxes and spectra and the X-ray luminosities for $B_{*} \sim 10-100 \mathrm{G}$; (2) the majority of stars have weaker fields so that the observed $\mathrm{X}$-rays are accompanied by a non thermal radio component too weak to be seen above the thermal flux; (3) X-rays come from a mixture of optically thin and optically thick emission so that the spectra do not show heavy photoelectric absorption; (4) the $\mathrm{X}$-ray spectra show no lines; (5) the W-R stars are soon likely to be detected as $\gamma$-ray sources; (6) if electrons are only injected in the inner wind acceleration region orders of magnitude more $\mathrm{X}$-rays and $\gamma$-rays than observed are produced. Where do the electrons come from ? From the same acceleration process operating in the solar-wind terrestrial magnetosphere interaction, in the sun, in SNRs, in radio galaxy jets, wherever a magnetised plasma is on the move? 